\title{
Controlled Active Thermal Storage in Smart PCM Walls for Energy Independent Building Applications
}

\author{
Richard Wegmueller, \\ ENERGY Institute, HEIA-FR \\ University of Applied Science of Western Switzerland, \\ HES-SO Fribourg \\ Fribourg, Switzerland \\ richard.wegmueller@hefr.ch \\ Dr. Jacques Robadey \\ ENERGY Institute, HEIA-FR \\ University of Applied Science of Western Switzerland, \\ HES-SO Fribourg \\ Fribourg, Switzerland \\ jacques.robadey@hefr.ch
}

\author{
Gabriel Magnin, \\ ENERGY Institute, HEIA-FR \\ University of Applied Science of Western Switzerland, \\ HES-SO Fribourg \\ Fribourg, Switzerland \\ gabriel.magnin@hefr.ch \\ Dr. Elena-Lavinia Niederhäuser \\ ENERGY Institute, HEIA-FR \\ University of Applied Science of Western Switzerland, \\ HES-SO Fribourg \\ Fribourg, Switzerland \\ elena-lavinia.niederhaeuser@hefr.ch
}

\begin{abstract}
The use of renewable energy sources towards autonomous buildings is promising, not only for electrical energy generation but also for heating purposes. However, it presents one fundamental issue: the shift between energy production and heating energy demand. This paper presents a solution to this problem in the form of an optimized, thermally controlled storage using phase change materials integrated to building walls. In contrast to the standard applications of phase change materials, which are currently used to solely improve the thermal inertia of buildings, this paper introduces an active management of heat storage. In particular, it allows the heat energy discharge through the activation of forced convection on demand. A test bench was designed and manufactured. Measurements performed showed excellent agreements with simulated results.
\end{abstract}

Keywords-heat storage; active management; phase change materials; autonomous buildings; numerical simulation; thermal; testbed; measurements

\section{INTRODUCTION}

Current solutions for energy storage include many disadvantages such as important financial expenses, large volumes, use of polluting materials (Li), not negligible energy losses and lack of dynamics during the restitution of energy. Indeed, an important time shift between heating demand and production is often remarked and it is a real problem, which explains why the improvement of thermal storage is a key solution to this problem Storing heat can be realized using phase change materials (PCM). These were originally utilized to increase comfort in the summer by avoiding overheating, the outside heat being trapped by the walls during the daytime and then released during the night. The suppression of heat peaks inside the buildings thanks to PCM leads also to economies in air conditioning. During winter, PCM walls are charged directly by the sun and discharged during the night. This is the case of the EMF Professional School building in Fribourg, Switzerland, where the thermal dynamics have been defined by a previous project.

The typical melting point of PCM used in buildings is between $19^{\circ} \mathrm{C}$ and $27^{\circ} \mathrm{C}$. Two groups of PCM are of interest for thermal regulation: hydrated salts, which however tend to supercool and may be chemically reactive, as well as paraffin wax, generally integrated into microcapsules. If PCM have an important storage capacity, reaching values from $1500 \mathrm{KJ} / \mathrm{m}^{2}$ [1] to $4000 \mathrm{KJ} / \mathrm{m}^{2}$ [2] for a $1 \mathrm{~cm}$ thick-plate, the main difficulty lies in their low heat conductivity $(\sim 0.15 \mathrm{~W} /(\mathrm{m} \cdot \mathrm{K}))$ limiting their power of charge and discharge.

In order to provide an achievable solution, the current paper proposes to use available PCM in an innovative way, introducing an active management of heat stocks and improving the dynamics of heat storage. Contrary to passive PCM, proactive management allows to use weather prediction data to accomplish energy savings, and also to fit dynamically heat restitution to the occupation of buildings. This is particularly interesting in the case of administrative and industrial buildings. The integration of PCM in adjustable walls increases the flexibility and the adaptability of the building, for a progressive urban use for medium to long term. Thanks to proactive management, storage can be accomplished according to weather predictions with energy savings up to 10$15 \%$. In addition, quick heat distribution can be made on request, thus guaranteeing comfort for the users. 
In this article, the design and operation of a test bench entirely developed and built at the ENERGY Institute - is firstly described before the presentation of the data acquisition procedure and the measurement results. A numerical model is also introduced in order to compare the simulated results with the measurements performed on the test bench.

\section{TEST BENCH}

To define the feasibility of the wall storage system, a representative test bench has been designed and built. It is characterized by two rooms of $2 \mathrm{~m}^{3}$ each, separated by a PCM wall and it is equipped with a heating system that uses a pumped hot water circuit to load the PCM plates. These ones are made of a mix of limestone and paraffin wax. The PCM low thermal conductivity is compensated by its integration in microcapsules, thus increasing the heat exchange surface. The main advantage of this implementation is that it presents no risk of liquid PCM leakage, since the paraffin is contained in a solid envelope. A 3D representation of the complete bench is showed in Fig. 1.

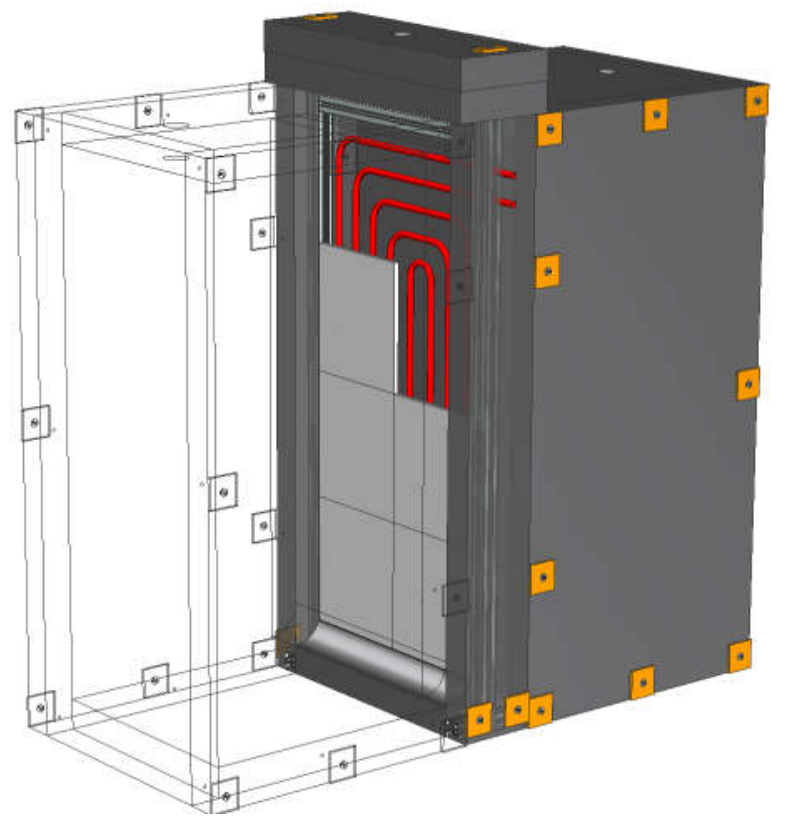

Fig. 1 Illustration of the test bench; heat exchanger represented in red, PCM plates in light gray

The heat restitution is controlled by forced convection. The PCM plates are separated from the wall insulation by an air layer of $10 \mathrm{~cm}$, which can be ventilated on request from the top to the bottom of the wall to extract the heat stored in the PCM.

In order to characterize the test bench and obtain quantitative results, the room and PCM plates temperature have been monitored. Thus, several sensors have been installed at specific places on the testbed displayed in Fig. 2, to provide representative values.

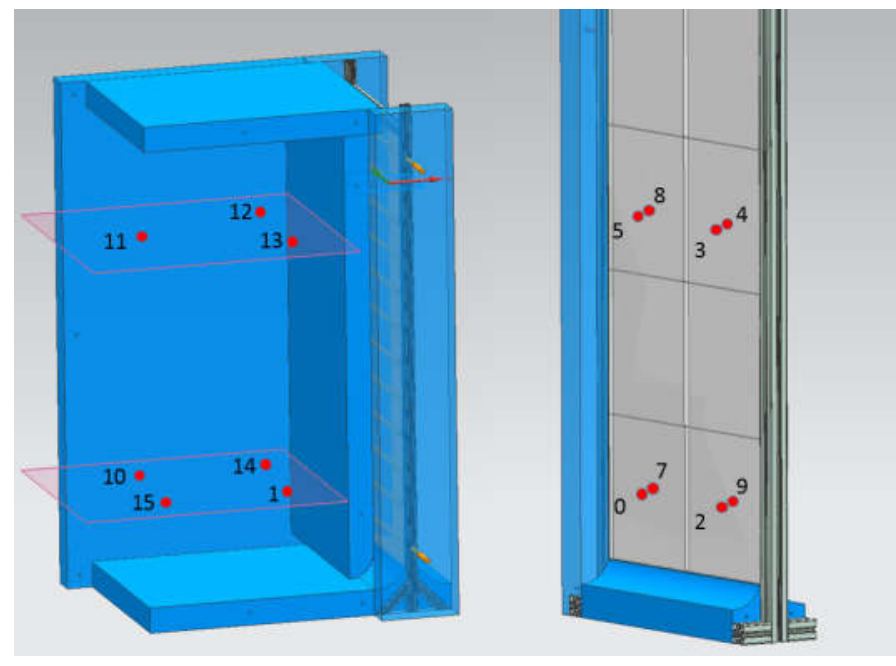

Fig. 2 Position of temperature sensors in the test bench

\section{MEASUREMENT PROTOCOL}

Measurements have been performed in order to characterize the capacity of PCM to store heat during whole night, before releasing it early in the morning. There were different testing phases: the first one corresponds to the loading phase during which water was heated (simulating a thermal solar panel energy source); the second phase is the storage phase and the last one the discharge phase. The measurements results of a two-day cycle are showed in Fig. 3 illustrating the variation in time of the mean temperature of the PCM plates, of the air in the rooms, the heating water and the external air. The loading phase lasted 6 hours while storage and discharge phases lasted 13 and 4 hours, respectively.

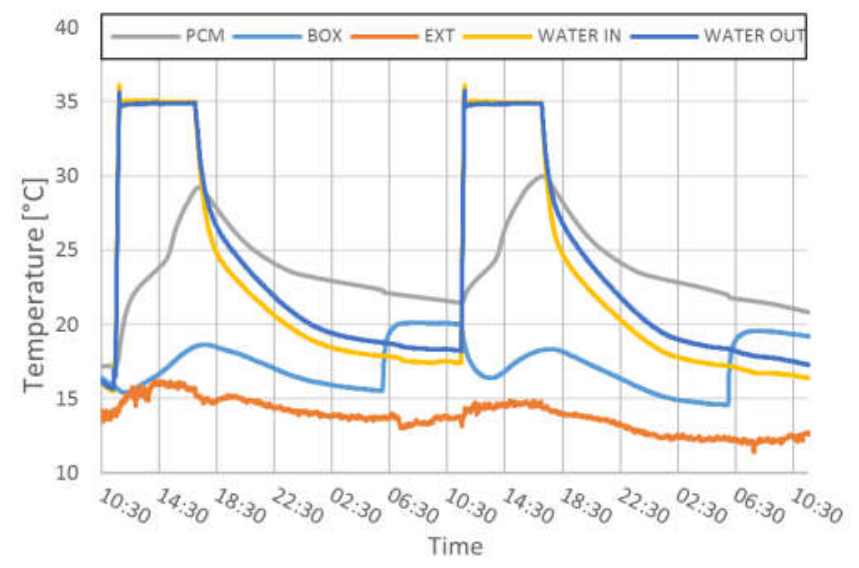

Fig. 3 Test bench measured temperatures for a two-day cycle

The measurements show that the storage in the PCM plates was sufficient to increase the temperature of the rooms from 15 to $20^{\circ} \mathrm{C}$. It is clearly visible, that the latent heat of the chosen material with a fusion temperature of $23^{\circ} \mathrm{C}$, greatly contributes to the heat storage. This effect is essential to guarantee a comfort air temperature inside the rooms. 


\section{NUMERICAL MODEL}

\section{A. Calculation Model}

In parallel to measurements, a numerical model was developed. The purpose is to establish a calculation model was to compare numerical results with the measured ones, to allow its application to a real size building. Simulations were performed using IDA ICE 4.7. This software allows the dynamic simulation of any building type and it was used in the present case, to model the test bench. The resolution of the energy balances in the different test bench zones allows to define temperatures in the different measurement points used during tests.

The most important parameter in this model is the PCM thermal capacity, with a specific heat assigned for every temperature level. As the PCM density of the PCM plates was known, the specific heat was estimated by interpolating the data from a known reference material (that has a PCM density 2.5 times lower than the used plates). This interpolation is presented in Fig. 4.

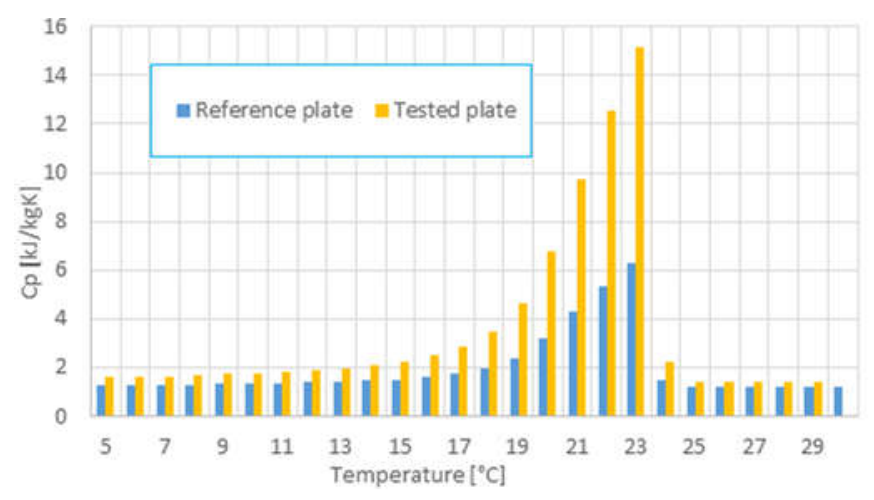

Fig. 4 Interpolation of specific heat values of PCM

If larger PCM concentrations increase thermal storage, the PCM plates can become brittle. One therefore has to find a compromise between storage capacity and assembly constraints.

\section{B. Simulation Results}

It is possible to obtain a variety of information from the simulations. In this study however, the only parameter treated is the temperature as it can be directly compared with the one obtained by measurements. Fig. 5 shows a typical simulation result for the temperatures of PCM plates, air in the rooms and outside air.

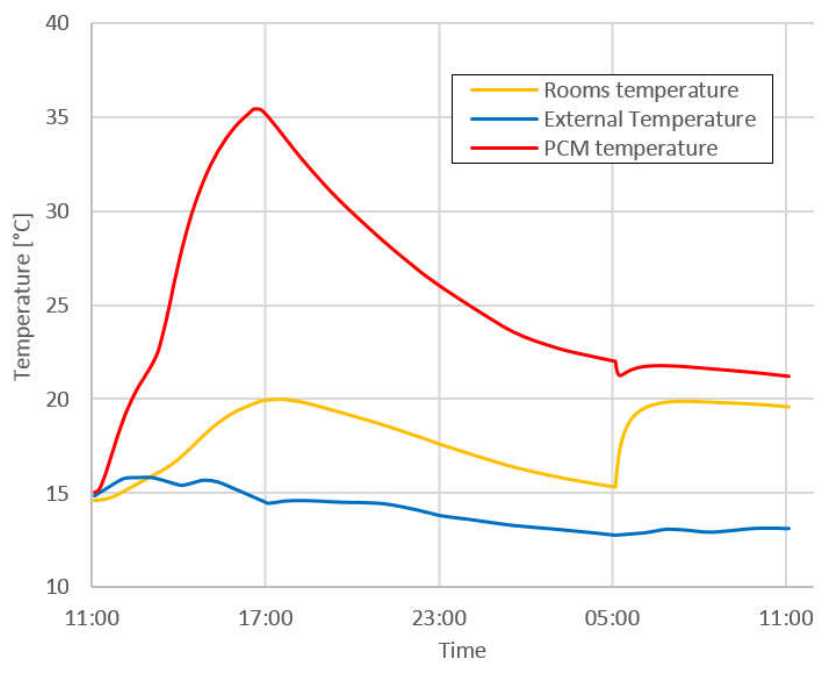

Fig. 5 Simulated temperatures during 24 hours

\section{RESUltS ANALYSIS}

A comparison between the measurements realized on the test bench (solid curves) and the simulated values (dashed curves) during 48 hours is shown in Fig. 6.

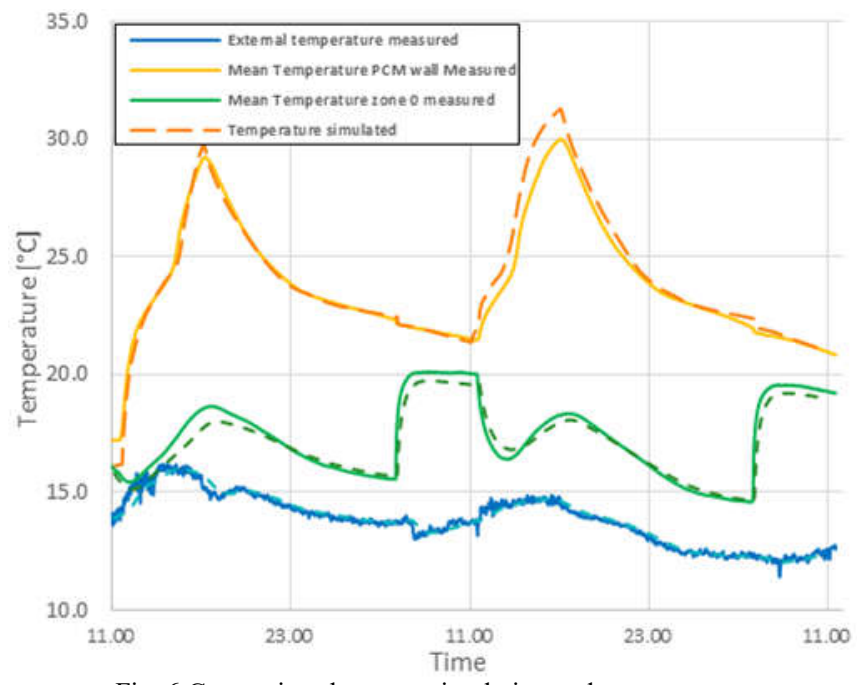

Fig. 6 Comparison between simulation and measurements

It can be seen that the PCM characterization by measurements and simulations is very similar and that the measurements results matches very well the simulation results. It is important to remind that the external temperature was an input of the simulation. The superposition of the blue curves is therefore perfect. A small discrepancy between the measured and simulated PCM temperatures is observed during the second day. One potential reason could be the use in the simulation of an overrated heat transfer coefficient between the heating system and the PCM. This value was fixed to $5 \mathrm{~W} /(\mathrm{m} 2 \cdot \mathrm{K})$, which is rather weak for a heat exchanger of this type but what corresponds to natural convection, a phenomenon very present all over the conception of the bench. 


\section{CONCLUSIONS}

This project allowed the validation of a new, innovative heat storage concept in buildings. Measurements and simulations results demonstrated the feasibility of the storage method with its energetical flexibility advantages. The test bench allowed obtaining interesting information about the possibilities to use isolated PCM panels inside building walls. The advantages of the heat storage using these materials were demonstrated through measurements realized on the test bench. The overnight latent heat storage allowed a rapid heating of the internal air of the box by $+5^{\circ} \mathrm{C}$ early in the morning. Thus, a comfort temperature was reached, despite the lack of heating during the entire night (10-12 hours). This illustrates the advantage of heat extraction by forced convection. Moreover that the test bench is also characterized by larger losses than a house or a standard building. The measurements are therefore very encouraging because one can expect even better thermal capacities if the presented concept is integrated in standard buildings. The material choice with a melting temperature at $23^{\circ} \mathrm{C}$ was purely arbitrary; it would be thus interesting to consider the same material with a melting point of $26^{\circ} \mathrm{C}$. This change could allow to reach higher PCM temperatures at the discharge time and thus increase the internal temperature of the tested room.

The numerical simulations can precisely demonstrate the feasibility of the concept in a building of standard size.

The comparison of the measures on the bench test and the results of the simulations allowed to validate the model created in IDA ICE. Thus, the same procedure can be used to redefine the concept for a standard building. The concept validation by measurements and numerical simulations could be obtained thanks to a relatively simple test bench with a single water circuit as heat source. No disturbances interfered with the measures and the deployment of the numerical model was therefore relatively simple. The same procedure will be applied in the future to simulate buildings of real sizes to validate the concept feasibility at large scale.

\section{REFERENCES}

[1] GLASSXcrystal, the glass that store heats and cools, Technical data, 2009

[2] Lemorange, Produktblatt Heizelement - Lehmwandheizung, 07.08.2013

[3] A. Carbonari, M. De Grassi, C. Di Perna, P. Pincipi, Numerical and experimental analyses of PCM containing sandwich panels for prefabricated walls, Energy and Buildings, 38, pp. 472-483, 2006.

[4] M. Ahmad, A. Bontemps, H. Sallée, D. Quenard, Thermal testing and numerical simulation of a prototype cell using light wallboards coupling vacuum isolation panels and phase change material, Energy and Buildings, 38, pp.673-681, 2006

[5] P.A. Galione, O. Lehmkuhl, J. Rigola, A. Oliva, I. Rodríguez, Numerical simulations of thermal energy storage systems with phase change materials, Universitat Politècnica de Catalunya, Termo Fluids S.L, Universidad de la República (Uruguay), 2010

[6] Didier Haillot, High performance storage composite for solar domestic hot water system enhancement : from raw material to the solar process, École doctorale Énergie environnement Perpignan (France), 2009

[7] Uros Stritih, An experimental study of enhanced heat transfer in rectangular PCM thermal storage, Faculty of Mechanical Engineering, University of Ljubljana (Slovenia), 2003

[8] Vineet Veer Tyagi, D. Buddhi, PCM thermal storage in buldings: A state of art, Thermal Energy Storage Laboratory, School of Energy \& Environmental Studies, Faculty of Engineering Science, Devi Ahilya University, Indore 452017, India, 2007

[9] C. Castellon, A. Castell, M. Martorelli and L. F. Cabeza ,Experimental Study of PCM Inclusion in Different Building Envelopes, Journal of Solar Energy Engineering, Volume 131, 2009

[10] Eva Günther, Stefan Hiebler, Harald Mehling, Robert Redlich, Enthalpy of Phase Change Materials as a Function of Temperature: Required Accuracy and Suitable Measurement Methods, International Journal of Thermophysics, 2009 